\title{
Efficient Room-Temperature Cooling with Magnets
}

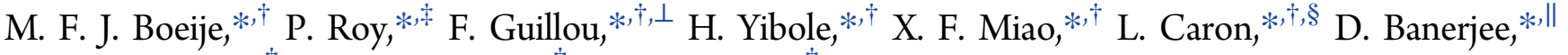 \\ N. H. van Dijk, ${ }^{* \dagger}$ R. A. de Groot, ${ }^{*}+{ }^{+}$and E. Brück ${ }^{*} \dagger$ \\ ${ }^{\dagger}$ Fundamental Aspects of Materials and Energy, Faculty of Applied Sciences, Delft University of Technology, Mekelweg 15, $2629 \mathrm{JB}$ \\ Delft, The Netherlands \\ ${ }^{\ddagger}$ Electronic Structure of Materials, Faculty of Sciences, Radboud University, 6525 AJ Nijmegen, The Netherlands \\ ${ }^{\perp}$ European Synchrotron Radiation Facility, 71 Avenue des Martyrs CS40220, F-38043 Grenoble Cedex 09, France \\ ${ }^{\S}$ Max Planck Institute for Chemical Physics of Solids, 01187 Dresden, Germany \\ "Dutch-Belgian Beamline (DUBBLE), ESRF - The European Synchrotron, CS 40220, 38043 Grenoble Cedex 9, France
}

\section{Supporting Information}

ABSTRACT: Magnetic cooling is a highly efficient refrigeration technique with the potential to replace the traditional vapor compression cycle. It is based on the magnetocaloric effect, which is associated with the temperature change of a material when placed in a magnetic field. We present experimental evidence for the origin of the giant entropy change found in the most promising materials, in the form of an electronic reconstruction caused by the competition between magnetism and bonding. The effect manifests itself as a redistribution of the electron density, which was measured by X-ray absorption and diffraction on $\mathrm{MnFe}(\mathrm{P}, \mathrm{Si}, \mathrm{B})$. The electronic redistribution is consistent with the formation of a covalent bond, resulting in a large drop in the $\mathrm{Fe}$ magnetic moments. The simultaneous change in bond length and strength, magnetism, and electron density provides the basis of the giant magnetocaloric effect. This new understanding of the mechanism of first order magneto-elastic phase transitions provides an essential step for new and improved magnetic refrigerants.

\section{INTRODUCTION}

Magnetic refrigeration based on the magnetocaloric effect (MCE) is a highly efficient cooling technology and the most promising alternative to gas compression-based refrigeration. Next to the perspective on improved efficiency compared to current vapor-compression technology it does not utilize greenhouse or ozone-layer depleting refrigerant gases, ${ }^{1}$ making it not only efficient but also environmentally friendly. The MCE is characterized by the isothermal entropy change and adiabatic temperature change resulting from a change in applied magnetic field. Under adiabatic conditions, the decrease in magnetic entropy due to moment alignment parallel to a magnetic field must be compensated by an increase in lattice entropy, leading to a temperature increase in the material. While this effect is intrinsic to all magnetic materials, near room temperature, one requires large magnetic fields, which are unpractical for applications.

However, materials such as $\mathrm{Mn}(\mathrm{As}, \mathrm{Sb}){ }^{2} \mathrm{La}(\mathrm{Fe}, \mathrm{Si})_{13}$ and its hydrides, ${ }^{3}$ and $(\mathrm{Mn}, \mathrm{Fe})_{2}(\mathrm{P}, \mathrm{X})$ where $\mathrm{X}=\mathrm{As},{ }^{4} \mathrm{Ge}^{5} \mathrm{Si}^{6}{ }^{6}$ $\mathrm{MnCoGe},{ }^{7}$ or $\mathrm{Gd}_{5}(\mathrm{Ge}, \mathrm{Si})_{4}{ }^{8}$ show a first-order magnetic transition, resulting in low field entropy changes that are 1 order of magnitude larger than for second-order transitions. Of these, the most promising magnetocaloric materials are the

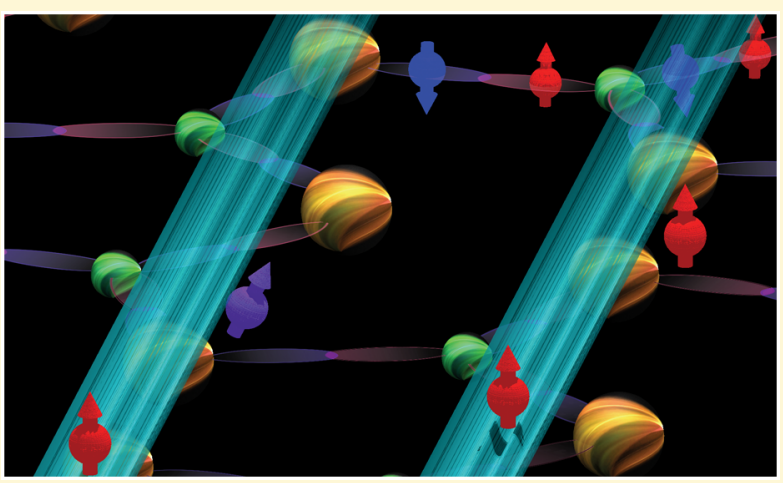

$\mathrm{Fe}_{2} \mathrm{P}$-based ${ }^{9}$ and $\mathrm{La}(\mathrm{Fe}, \mathrm{Si})_{13}$-based $^{3}$ compounds. They show a large isothermal entropy change and adiabatic temperature change in low magnetic fields in combination with a small thermal hysteresis. In addition, they both exhibit a magnetoelastic transition, in which the atomic arrangement changes in a much more subtle way compared to the full crystallographic phase transition encountered in other first-order materials.

Previous density functional theory (DFT) calculations predict a distinct change in electronic structure and magnetic moments across the magneto-elastic transition in $\mathrm{Fe}_{2} \mathrm{P}$-based materials. ${ }^{10-12}$ In particular, $\mathrm{Fe}$ atoms on the $3 f$ site display a partially quenched magnetic moment in the paramagnetic state. (crystal structure in Supporting Information). A recent DFT study on $\mathrm{La}(\mathrm{Fe}, \mathrm{Si})_{13}$-based compounds also indicates, for the paramagnetic state, "the possibility of stable quenched Femoments". ${ }^{13}$ We therefore propose that competition between bond formation and moment formation (cartoon in Figure 1) lies at the basis of this first-order magnetoelastic transition as it involves the same $3 d$ electrons.

Received: February 4, 2016

Revised: June 6, 2016

Published: June 6, 2016 


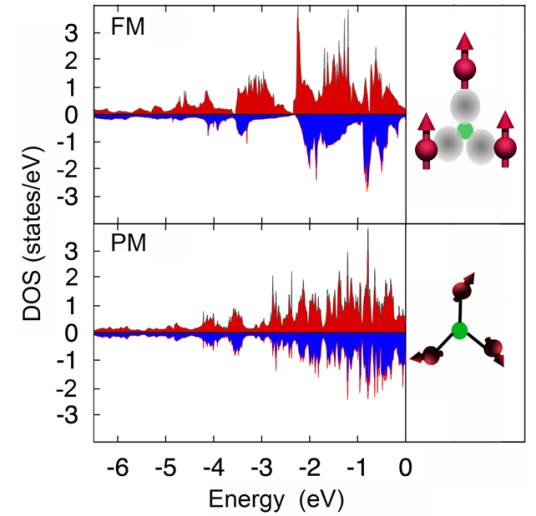

Figure 1. Partial density of states of Fe in the ferromagnetic (FM) and the paramagnetic (PM) phase of $\mathrm{MnFeP}_{0.67} \mathrm{Si}_{0.33}$ obtained by DFT calculations. The energy scale is plotted with respect to the Fermi energy. In the ferromagnetic phase there is a majority of spin-up electrons (red) compared to the spin-down electrons (blue), which contribute to the itinerant magnetism $\left(1.45 \mu_{\mathrm{B}}\right)$. In the paramagnetic phase there is less spin polarization, which results in smaller magnetic moments $\left(0.67 \mu_{\mathrm{B}}\right)$. The latter is due to the formation of covalent bonds between $\mathrm{Fe}$ and $\mathrm{P} / \mathrm{Si}$ as depicted in the cartoon on the right panel.

In order to experimentally verify that this mechanism is relevant in MCE materials, one may probe changes in electron density around the $\mathrm{Fe}$ atoms. The $\mathrm{MnFe}_{0.95} \mathrm{P}_{0.582} \mathrm{Si}_{0.34} \mathrm{~B}_{0.078}{ }_{14}$ compound was chosen for its sharp first-order transition and an $\mathrm{Fe} / \mathrm{Mn}$ ratio close to $1: 1$, which allows one to distinguish the high and low moment sites. For $\mathrm{La}(\mathrm{Fe}, \mathrm{Si})_{13}$ experimental verification is more difficult as all moments are $\mathrm{Fe}$ moments. We use X-ray absorption fine structure (XAFS) and highresolution X-ray diffraction (XRD) to investigate the electron density. Extended X-ray absorption fine structure (EXAFS) is sensitive to the local electronic environment around a particular type of atom. By applying a Fourier transform to the EXAFS signal, 1D radial electron density plots can be obtained. By measuring below and above the critical temperature, changes in this radial distribution of the electron density are detected. To characterize these changes also in $3 \mathrm{D}$, we created charge density maps from high-resolution XRD measurements above and below the critical temperature. Additionally, we performed new DFT calculations for a compound with a stoichiometry close to the experimental value.

\section{EXPERIMENTAL SECTION}

Preparation of the sample is described in a previous paper. ${ }^{14}$ The X-ray absorption and powder diffraction experiments were performed at the DUBBLE $^{15}$ (BM26A) and BM1A beamlines, respectively, both located at the ESRF in Grenoble.

For the absorption experiments, powders with particle sizes between 20 and $40 \mu \mathrm{m}$ were mixed with boron nitride and pressed into pellets. The measurements were performed in transmission mode. Boron nitride secures the structural properties of the material without influencing the measurement. A nitrogen cryostream was used to cool and heat the samples with a temperature control within $2 \mathrm{~K}$. For better statistics, two absorption spectra were recorded at each temperature.

For the diffraction experiments, powder from the same batch as the absorption experiments was loaded in $0.7 \mathrm{~mm}$ capillaries and measured at four temperatures $(150,250,293$, and $350 \mathrm{~K})$ again using a nitrogen cryostream. A wavelength of $0.6963 \AA$ was used and angles up to $2 \theta=$ $57^{\circ}$ were measured, yielding about 80 reflections. The data reduction and details of the DFT calculations are described in the Supporting Information.

\section{RESULTS AND DISCUSSION}

As shown in Figure 1 for $\mathrm{MnFeP}_{0.67} \mathrm{Si}_{0.33}$ a reduction in magnetic moments of the $\mathrm{Fe}$ atoms is found in our new DFT calculations (details on the method and the corresponding data for $\mathrm{Mn}$ can be found in the Supporting Information). At the same time, the local charge distribution around the $\mathrm{Fe}$ atoms drastically changes at the ferromagnetic transition.

In Figure 2 the Fourier transform of the Fe EXAFS data, measured below and above $T_{C}$, is shown as a function of the

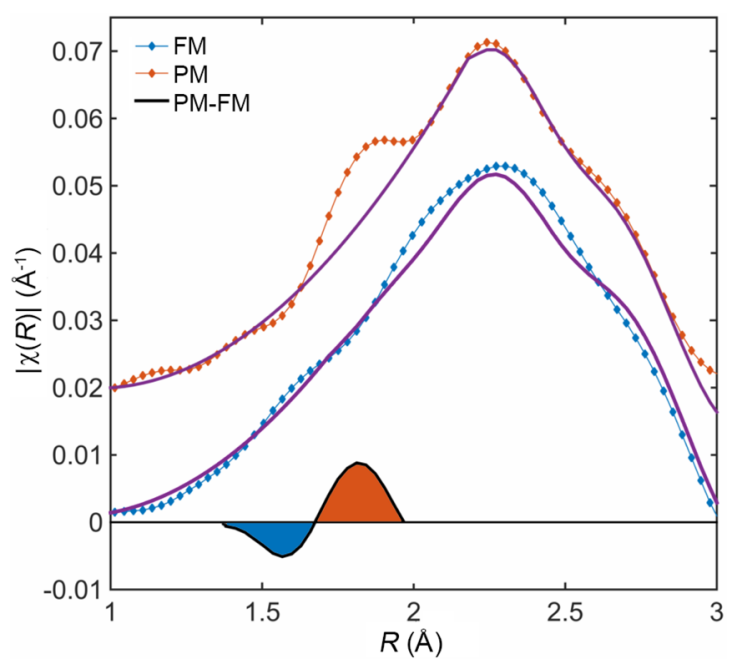

Figure 2. Radial plots of the electron density around the Fe atom as derived from EXAFS in the FM phase (measured at $100 \mathrm{~K}$ ) and in the PM phase (measured at $400 \mathrm{~K}$ ). The PM curves have been shifted vertically by $0.02 \AA^{-1}$ for clarity. The connected points represent the measured data, and the solid lines are fits to the data based on the atomic positions using an isotropic model. The black solid line represents the difference between the FM and PM phase.

distance from the central absorber atom. As X-rays solely interact with electrons, this represents the radial distribution of the electron density around Fe. Two electron density peaks around 2.3 and $2.7 \AA$ are caused by atoms occupying the $1 b / 2 c$ and $3 f / 3 g$ positions. We know from neutron diffraction that the $1 b$ and $2 c$ sites are occupied by $\mathrm{P}$ and $\mathrm{Si}$ atoms, while $\mathrm{Fe}$ and $\mathrm{Mn}$ atoms occupy the $3 f$ and $3 g$ positions. ${ }^{16}$ A small shift in peak position reflects the difference in interatomic distances for the FM and the PM phase. In addition to the main two peaks, another peak is visible located at smaller distances. This peak is located at distances of 1.8 and $1.6 \AA$ above and below $T_{\mathrm{C}}$, respectively. Therefore, our simulations based on the atomic positions obtained from neutron diffraction clearly reproduce the $1 b / 2 c$ and $3 f / 3 g$ peaks but are not able to reproduce the first peak. Note that the position of the Fe absorption edge is not affected, which excludes a change in $\mathrm{Fe}$ valence. The EXAFS results are a first indication that the Fe electron charge is indeed redistributed locally across the transition.

While the radially averaged electron density is probed by element-specific EXAFS, it can be probed in 3D by highresolution X-ray diffraction. The inverse Fourier transform of the structure factors, as determined by Rietveld refinement, ${ }^{17}$ can be used to experimentally obtain a 3D electron density plot, analogous to that obtained from DFT calculations.

As we are interested in the changes in electron density related to the magnetoelastic phase transition, we subtract the electron densities of the ferromagnetic from the paramagnetic 


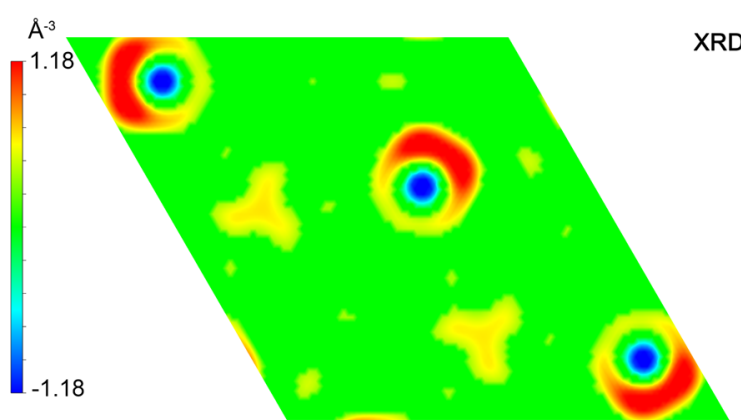

(a)

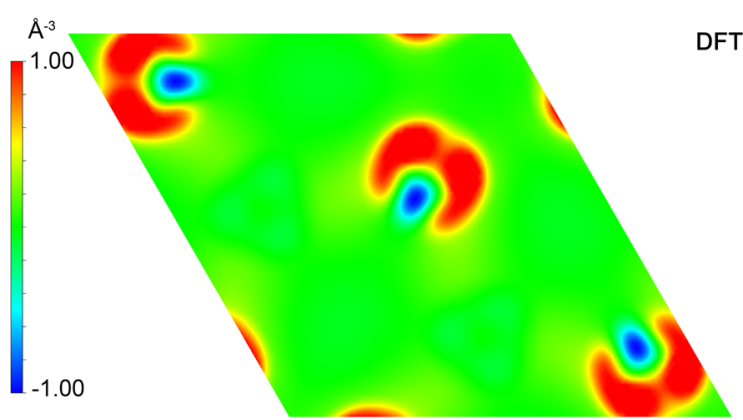

(b)

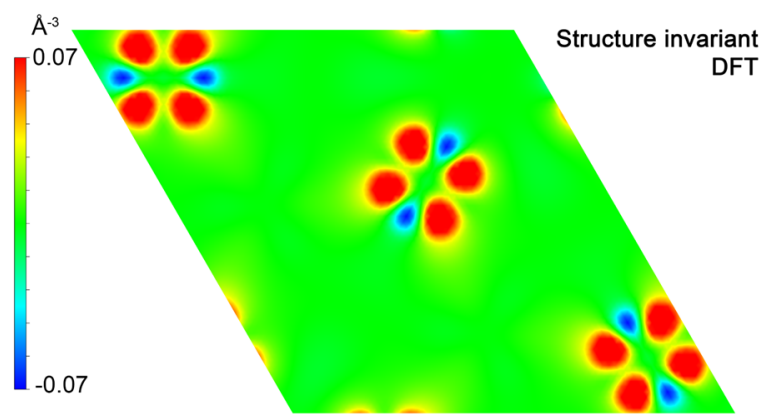

(c)

Figure 3. Charge difference map across the ferromagnetic transition from high-resolution X-ray powder diffraction at 350 and $150 \mathrm{~K}$ (a) and DFT calculations $(\mathrm{b}$ and $\mathrm{c})$. The unit cells were uniformly scaled when subtracting the phases. Structure invariant indicates that the relative Fe coordinate was fixed. The difference between the FM state (blue) and the PM state (red) is shown.

phase. The difference maps are shown in Figure 3, in particular for the electron density difference within the Fe layer. Figure 3a was generated by subtracting the measured electron density at $150 \mathrm{~K}$ from the measured electron density at $450 \mathrm{~K}$. Parts b and c of Figure 3 are both derived from first principle DFT calculations. In the case of Figure $3 \mathrm{~b}$ the $\mathrm{Fe}$ atoms are allowed to relax to the experimentally determined equilibrium position, while for Figure $3 c$ the relative coordinates of the atoms were kept fixed.

The calculated (Figure 3b) and experimentally determined (Figure 3a) electron density difference plots show very similar features around Fe. The redistribution of electron density is asymmetric due to a shift of the atomic position of $\mathrm{Fe}$ across the transition. Both the experimental shift and the lattice parameters were used as input for the calculations in order to reliably compare the two density plots. Similar calculations are performed to generate Figure 3c, however keeping the relative atomic positions constant. ${ }^{18}$ It shows that electrons shift from a $d_{x z}$ to a $d_{z}{ }^{2}$ orbital when crossing the magnetoelastic transition from the paramagnetic to the ferromagnetic state, respectively. In Figure $3 \mathrm{~b}$ one lobe of the $d_{z}{ }^{2}$ orbital is obscured, and the two lobes of the $d_{x z}$ orbital on either side of the atom are smeared out.

The asymmetry that is observed in the direct subtraction can be reduced if one switches to radial coordinates. By taking the atomic coordinates of $\mathrm{Fe}$ and summing up all charges found at certain distance intervals we generate radial electron-density plots from the experimental XRD data at 150 and $350 \mathrm{~K}$ (see Supporting Information). This radial electron-density plot reproduces the unidentified peak and peak position that were observed in Figure 2 at short distance and the difference plot shows the same behavior as that found in the EXAFS results. XRD and EXAFS thus corroborate the earlier statement that was based on DFT calculations; in these materials moment formation competes with bonding.

In the PM phase, a covalent bond is formed between $\mathrm{Fe}$ and the $\mathrm{P} / \mathrm{Si}$ atoms, leading to a decrease in the $a / b$ and an increase in the $c$ lattice parameters. This can be understood from a magnetic and a chemical viewpoint. The Fe atoms only carry a sizable magnetic moment when the exchange interaction between the Mn layers is sufficiently strong. However, as the exchange interaction is overcome by magnetic fluctuations, we find that the ferromagnetic transition is associated with a remarkably strong reduction of the $\mathrm{Fe}$ magnetic moment. In other words, the ferromagnetic exchange field exerted by the $\mathrm{Mn}$ moments on the $\mathrm{Fe}$ positions stabilizes the $\mathrm{Fe}$ magnetic moments, while as soon as it vanishes, the $\mathrm{Fe}$ valence electrons rather bond with neighboring $\mathrm{P} / \mathrm{Si}$, which destabilizes the moments.

The experimental correspondence with the DFT calculations indicates that the metalloid atoms play a crucial role in providing metastable behavior in these compounds, namely that the electrons can switch between metallic and covalent character. Metalloids share properties of both metals and nonmetals. This was recognized early on by Linus Pauling in his influential work on the chemical bond by looking at the crystal structures of $\mathrm{C}, \mathrm{Si}, \mathrm{Ge}$, and $\mathrm{Sn} .{ }^{19}$ Carbon, being small, has a large overlap of its orbitals and prefers to make directional, localized bonds. For Si and Ge the overlap decreases, and for Sn the overlap is so small that, above room temperature, the diamond structure is transformed into a metallic phase. The metastability in bonding due to metalloids is the basis for many first-order magnetostructural transitions, and for this system it is found to be responsible for the magnetoelastic isostructural transition. 


\section{CONCLUSIONS}

In summary, we observe a redistribution in the electron density around the $\mathrm{Fe}$ atom when going from the FM to the PM phase by XAFS and XRD measurements. This results in a formation of covalent bonds that strongly reduce the magnetic moment of Fe. Therefore, mixed magnetism is demonstrated to be the underlying phenomenon responsible for the first-order magnetic phase transition and the excellent magnetocaloric properties found in $\mathrm{Fe}_{2} \mathrm{P}$-based compounds.

Besides the magnetic (order-disorder) and crystallographic changes, there is also a distinct electronic contribution to the entropy change, which allows tapping into all three entropy reservoirs (lattice, magnetic, and electronic contributions) of the material. Apart from the early work on FeRh, ${ }^{20}$ the electronic contribution to the magnetocaloric effect is usually disregarded or considered to be negligible. The electronic contribution arises from the redistribution of electrons and a change in density of states at the Fermi level, of which Fe shows the largest contribution.

This effectively boosts the magnetocaloric effect, as the entropy change relies not only on the Mn layer moment alignment but also on the change in the electron density of states in the $\mathrm{Fe}$ layer. Its relevance becomes strikingly clear when one considers the temperature scales of each contribution. Applied magnetic fields of a few tesla correspond to a few kelvin, while the ordering of the magnetic moments is characterized by the $T_{\mathrm{C}} \approx 300 \mathrm{~K}$. The structural contribution is characterized by the Debye temperature for lattice vibrations $\Theta_{\mathrm{D}} \approx 420 \mathrm{~K}$ for $\mathrm{Fe}_{2} \mathrm{P}$. The electronic contribution, however, can reach up to the Fermi temperature $T_{\mathrm{F}} \approx 10000 \mathrm{~K}$.

The reduction in the $\mathrm{Fe}$ moment on only one crystallographic site in $\mathrm{MnFe}(\mathrm{P}, \mathrm{Si})$ and $\mathrm{La}(\mathrm{Fe}, \mathrm{Si})_{13}$ indicates that both materials behave similarly. Now that the origin of the giant magnetocaloric effect for these materials is established, we propose two minimal requirements for possible candidate materials with further improved magnetocaloric properties. These materials should contain a metalloid element and a late $3 d$ transition metal that occupies two different crystallographic positions. These ingredients provide the necessary tools to design mixed magnets for cooling applications with improved efficiencies.

\section{ASSOCIATED CONTENT}

\section{S Supporting Information}

The Supporting Information is available free of charge on the ACS Publications website at DOI: 10.1021/acs.chemmater.6b00518.

The density of states calculations for $\mathrm{Fe}$ and $\mathrm{Mn}$, the XAFS data, the XRD refinement parameters, and electron density plots (PDF)

\section{AUTHOR INFORMATION}

\section{Corresponding Authors}

*(M.F.J.B.) E-mail: m.f.j.boeije@tudelft.nl.

*(P.R.) E-mail: p.roy@science.ru.nl.

*(F.G.) E-mail: francois.guillou@esrf.fr.

*(H.Y.) E-mail: yibole@tudelft.nl.

*(X.F.M.) E-mail: x.f.miao@tudelft.nl.

*(L.C.) E-mail: caron@cpfs.mpg.de.

*(D.B.) E-mail: dipanjan.banerjee@esrf.fr.

*(N.H.v.D.) E-mail: n.h.vandijk@tudelft.nl.

*(R.A.d.G.) E-mail: r.degroot@science.ru.nl.
*(E.B.) E-mail: e.h.bruck@tudelft.nl.

\section{Notes}

The authors declare no competing financial interest.

\section{ACKNOWLEDGMENTS}

This work is financially supported by the Foundation for Fundamental Research on Matter (FOM) under IPP-i28 and is cofinanced by BASF New Business. We acknowledge the European Synchrotron Radiation Facility for provision of synchrotron radiation facilities and thank the beamline staff for assistance in using the DUBBLE beamtime and beamline BM1A. We like to thank Alex Brownrigg for his assistance with the sample preparation and Philip Pattison for performing the high resolution XRD measurements.

\section{REFERENCES}

(1) Gutfleisch, O.; Willard, M. A.; Brück, E.; Chen, C.; Sankar, S.; Liu, J. P. Magnetic Materials and Devices for the 21st Century: Stronger, Lighter, and More Energy Efficient. Adv. Mater. 2011, 23, $821-842$.

(2) Wada, H.; Tanabe, Y. Giant magnetocaloric effect of $\mathrm{MnAs}_{1-x} \mathrm{Sb}_{x}$. Appl. Phys. Lett. 2001, 79, 3302-3304.

(3) Fujita, A.; Fujieda, S.; Hasegawa, Y.; Fukamichi, K. Itinerantelectron metamagnetic transition and large magnetocaloric effects in $\left.\mathrm{LaFe}_{x} \mathrm{Si}_{1-x}\right)_{13}$ compounds and their hydrides. Phys. Rev. B: Condens. Matter Mater. Phys. 2003, 67, 104416.

(4) Brück, E.; Ilyn, M.; Tishin, A.; Tegus, O. Magnetocaloric effects in $\mathrm{MnFeP}_{1-x} \mathrm{As}_{x}$-based compounds. J. Magn. Magn. Mater. 2005, 290291, 8-13.

(5) Trung, N.; Ou, Z.; Gortenmulder, T.; Tegus, O.; Buschow, K.; Brück, E. Tunable thermal hysteresis in $\operatorname{MnFe}(\mathrm{P}, \mathrm{Ge})$ compounds. Appl. Phys. Lett. 2009, 94, 102513.

(6) Tegus, O.; Brück, E.; Buschow, K. H. J.; de Boer, F. R. Transition-metal-based magnetic refrigerants for room-temperature applications. Nature 2002, 415, 150-152.

(7) Trung, N. T.; Zhang, L.; Caron, L.; Buschow, K. H. J.; Brück, E. Giant magnetocaloric effects by tailoring the phase transitions. Appl. Phys. Lett. 2010, 96, 172504.

(8) Pecharsky, V. K.; Gschneidner, K. A., Jr. Giant magnetocaloric effect in $\mathrm{Gd}_{5} \mathrm{Si}_{2} \mathrm{Ge}_{2}$. Phys. Rev. Lett. 1997, 78, 4494-4497.

(9) Lundgren, L.; Tarmohamed, G.; Beckman, O.; Carlsson, B.; Rundqvist, S. First Order Magnetic Phase Transition in $\mathrm{Fe}_{2}$ P. Phys. Scr. 1978, 17, 39-48.

(10) Yamada, H.; Terao, K. First-order transition of Fe2P and antimetamagnetic transition. Phase Transitions 2002, 75, 231-242.

(11) Delczeg-Czirjak, E. K.; Delczeg, L.; Punkkinen, M. P. J.; Johansson, B.; Eriksson, O.; Vitos, L. Ab initio study of structural and magnetic properties of Si-doped $\mathrm{Fe}_{2} \mathrm{P}$. Phys. Rev. B: Condens. Matter Mater. Phys. 2010, 82, 085103.

(12) Dung, N. H.; Ou, Z. Q.; Caron, L.; Zhang, L.; Thanh, D. T. C.; de Wijs, G. A.; de Groot, R. A.; Buschow, K. H. J.; Brück, E. Mixed Magnetism for Refrigeration and Energy Conversion. Adv. Energy Mater. 2011, 1, 1215-1219.

(13) Gruner, M.; Keune, W.; Cuenya, B.; Weis, C.; Landers, J.; Makarov, S.; Klar, D.; Hu, M.; Alp, E.; Zhao, J.; Krautz, M.; Gutfleisch, O.; Wende, H. Element-Resolved Thermodynamics of Magnetocaloric $\mathrm{LaFe}_{13-x} \mathrm{Si}_{x^{*}}$. Phys. Rev. Lett. 2015, 114, 057202.

(14) Guillou, F.; Porcari, G.; Yibole, H.; van Dijk, N. H.; Brück, E. Taming the first-order transition in giant magnetocaloric materials. Adv. Mater. 2014, 26, 2671-2675.

(15) Nikitenko, S.; Beale, A. M.; van der Eerden, A. M. J.; Leynaud, S. D. M. J. A. O.; O’Brien, M. G.; Detollenaere, D.; Kaptein, R; Weckhuysen, B. M.; Bras, W.; Jacques, S. D. M. Implementation of a combined SAXS/WAXS/QuEXAFS set-up for time-resolved in situ experiments. J. Synchrotron Radiat. 2008, 15, 632-640. 
(16) Miao, X. F.; Caron, L.; Gercsi, Z.; Daoud-Aladine, A.; van Dijk, N. H.; Brück, E. Thermal-history dependent magnetoelastic transition in $(\mathrm{Mn}, \mathrm{Fe})_{2}(\mathrm{P}, \mathrm{Si})$. Appl. Phys. Lett. 2015, 107, 042403.

(17) Rietveld, H. M. A profile refinement method for nuclear and magnetic structures. J. Appl. Crystallogr. 1969, 2, 65-71.

(18) The scale of Figure $3 \mathrm{c}$ is 1 order of magnitude lower compared to the scale of Figure $3 \mathrm{~b}$. This difference can be attributed to the use of the experimental crystal parameters.

(19) Pauling, L. The nature of the chemical bond; Cornell University Press: 1960 .

(20) Annaorazov, M. P.; Nikitin, S. A.; Tyurin, A. L.; Asatryan, K. A.; Dovletov, A. K. Anomalously high entropy change in FeRh alloy. J. Appl. Phys. 1996, 79, 1689-1695. 